\title{
AXONAL PATHFINDING IN THE ABSENCE OF NORMAL PATHWAYS AND IMPULSE ACTIVITY ${ }^{1}$
}

\author{
WILLIAM A. HARRIS \\ Department of Biology, University of California, San Diego, La Jolla, Califoria 92093
}

Received September 2, 1983; Revised December 13, 1983; Accepted December 22, 1983

\begin{abstract}
Retinal axons were challenged to grow to their targets both along abnormal pathways and in the absence of impulse activity. Eye primordia were first transplanted from normal to ectopic sites in axolotl embryos. Most of the hosts were genetically eyeless, others were enucleated normal embryos. These axolotl embryos were then parabiotically joined to California newt embryos. Both operations were completed by stage 28 , which is before axons have left the eye. The result of the parabiosis was a paralysis of the "eyeless" axolotl twin due to the newt's tetrodotoxin (TTX), while the newt twin remained normally active. When the axolotl twin reached early larval stage, about 1 week later, the projection from the silent transplanted retina was assessed using horseradish peroxidase (HRP) injections into the retina, after which the animals were killed and prepared histologically to reveal the presence of HRP in neuronal processes. The results from 17 such cases show normal topographic retinotectal projections: the dorsal retina projecting to the ventrolateral tectum, and the ventral retina projecting to the dorsomedial tectum. Unusual pathways were often taken to achieve these destinations. Control animals, both normal axolotl larvae developing alone and normal axolotl larvae parabiosed to newts, also showed the normal retinotectal projection patterns. These results indicate that the retinal projections in the experimental group were basically normal.

Thus, fibers need neither impulse activity nor a particular pathway to navigate to their correct targets during development. Both factors can be eliminated simultaneously, yet retinal axons still find their way to the tectum and make an ordered map. This indicates that other factors, such as the chemoaffinity mechanisms proposed by Sperry (Sperry, R. W. (1963) Proc. Natl. Acad. Sci. U. S. A. 50: 703-710), may play a more major role in axonal pathfinding in this system.
\end{abstract}

An axon's growth to its proper target appears to be a complex phenomenon (see recent review by Letourneau, 1982). Observations of normal development and experiments in developing and regenerating systems have provided evidence for several different mechanisms at work in the process of axonal navigation. In lower vertebrates, studies of the central visual projection from the retina to the tectum have been particularly informative. However, results from this system, may at first seem confusing and contradictory (for reviews see Gaze, 1970; Horder and Martin, 1978; Jacobson, 1978; Fraser and Hunt,

${ }^{1}$ This work was supported by National Institutes of Health Grant HD 14490, a March of Dimes Basil O'Connor grant, and the McKnight Foundation. Nancy Gallenson provided expert technical help. I thank her, as well as Max Cowan, Steve Easter, James Fawcett, Betty Ferguson, Scott Fraser, Jeff Hall, Christine Holt, Janet Lamborghini, and Barbara Taylor for helpful discussions and comments on the manuscript.
1980). For example, retinal fibers normally grow along particular pathways to the tectum and can be shown to favor these pathways (Harris, 1980b, 1982; Gaze and Fawcett, 1982), yet retinal fibers may also successfully grow to the tectum if forced to use other pathways (Hibbard, 1967; Harris, 1982). Another example is that retinal fibers appear to need impulse activity to form a finely tuned map of visual space on the tectum (Schmidt and Fdwards, 1982; Meyer, 1983), yet they do not need impulses either to reach their target neuropil or to make at least a rough topographic map (Harris, 1980a; Schmidt and Edwards, 1982; Meyer, 1983). Developing fibers actively fasciculate into brachia that lead retinal axons to the appropriate tectal sectors (Gaze and Fawcett, 1982; Fawcett and Gaze, 1982; Easter, 1983), yet regenerating and developing fibers may reach their targets without traveling in any particular fascicles or brachia (Fujisawa et al., 1982; Harris, 1982). Fibers grow out of the retina in a temporal sequence that could serve to assist them 
in the formation of a normal topographic projection (Bunt and Horder, 1977; Scholes, 1979; Bodick and Levinthal, 1980; Easter, 1983; Holt, 1984). However, the temporal sequence can be distributed without appreciable consequence to the map (Feldman et al., 1971; Holt, 1984). Finally, fibers from a half retina will spread over an entire tectum showing that repulsive interactions between fiber terminals are involved in map formation (Schmidt et al., 1978; Straznicky et al., 1981; Schmidt, 1982 ), yet when half eye or compound eye projections are first formed they are restricted to their appropriate topographic locations (Straznicky et al., 1981; Ferguson, 1983). When stated in this way, the above results can be seen to imply that many mechanisms may be used, but none of the ones tested is solely required. Mechanical, electrical, fiber-interactive, and temporal mechanisms may all play some role in the development of neuronal connections. However, each of these factors may be interfered with or inhibited in some way, without preventing relatively accurate axonal navigation within the central nervous system.

The role of "chemoaffinity" (Sperry, 1963) in the development of retinotectal connections is as yet unclear. Recently there have been convincing demonstrations in vitro of affinities between growing retinal axons and tectal cells (Bonhoeffer and Huf, 1982), even tectal cell membranes (IIalfter et al., 1981; B. Kern-Veits and F. Bonhoeffer, submitted for publication). These affinities are arranged in an appropriate gradient for normal map formation. A strong gradient of a cell surface antigen on the retina has also been found (Trisler et al., 1981). It may well be true that certain cytochemical factors are involved in normal axonal navigation, either as the molecular basis for some of the above mechanisms or in addition to them. Since axons may use more than one cue at a time, eliminating a single such factor might not prevent relatively accurate navigation within the central nervous system.

The lesson learned from studies of pigeon homing, that pigeons can orient properly either on a cloudy day or with an abnormal magnetic field (Keeton, 1974), applies to axonal "homing" as well. Pigeons in fact use both the sun and magnetism to orient. Disorientation occurs only when both cues are subverted in combination. Might axons also have many different cues available to them for navigation? If so, a good way to judge the relative importance or unimportance of certain mechanisms would be to interfere with them combinatorially. 'This paper represents a beginning of such an approach. Developing retinal axons in vivo have been forced to grow both in the absence of impulse activity and along abnormal pathways. This has been done hy combining two previous experiments: forcing the fibers to grow along an abnormal pathway by grafting an optic vesicle from a normal to an ectopic region in an eyeless axolotl (Schwenk and Hibbard, 1977; Harris, 1982), and eliminating impulse activity by parabiosing the operated axolotl to the tetrodotoxin (TTX)-harboring California newt (Twitty, 1937; Harris, 1980a). The findings presented here, that retinal axons grow to their appropriate region of the tectum in the absence of impulse activity and take abnormal trajectories, rule out this one combi- nation of cues as critical for axonal navigation and target selection and opens the way for more comprehensive combinatorial experiments.

\section{Materials and Methods}

Embryonic surgery. Embryonic operations were done in two steps (see Fig. 1). In the first, eye primordia were transplanted from normal to eyeless axolotl embryos at stages 24 to 26 (Schreckenburg and Jacobson, 1975), following previous methods (Harris, 1982). Axolotl embryos from a spawning of adults, each hetcrozygous for the eyeless mutation $(e /+)$, were sorted into presumptive phenotypic mutants (e/e) and presumptive normal embryos $(e /+$ and $+/+)$. Eye development is halted at a very early stage in these mutants, because the optic cup does not evaginate from the diencephalic neuroepithelium, there is no optic stalk and no primordial retina

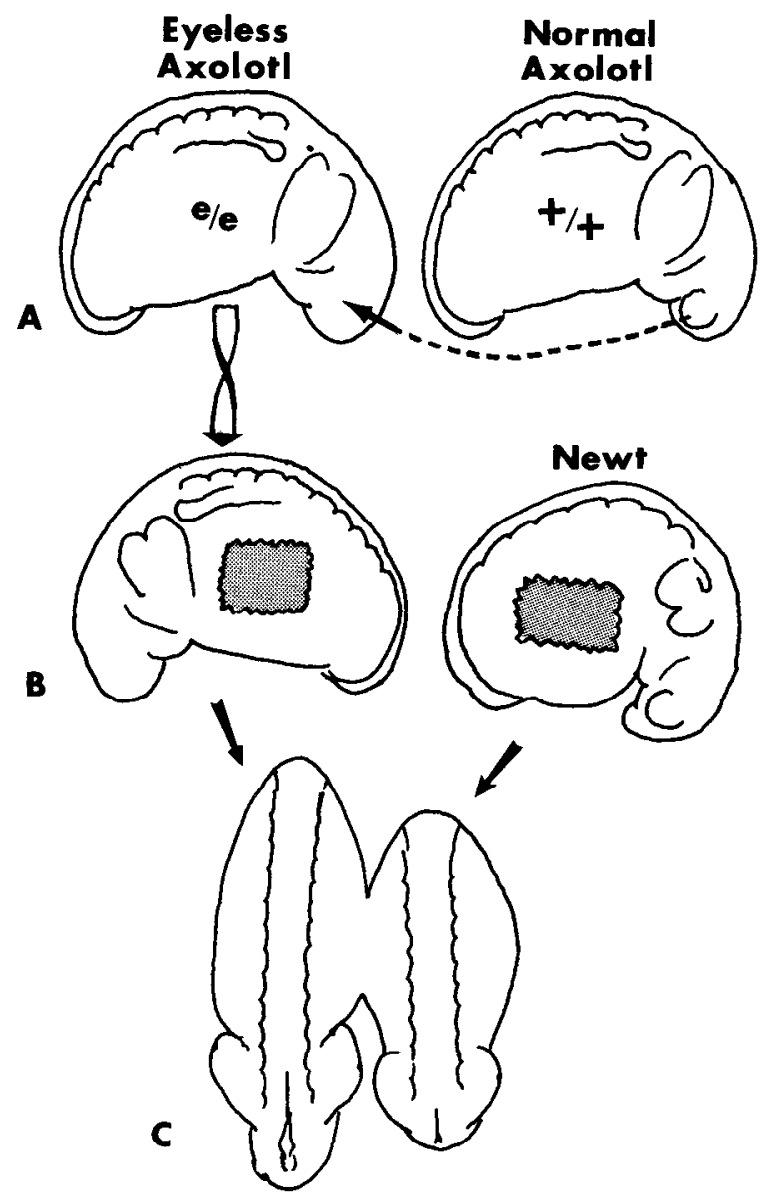

Figure 1. 'I'ransplantation and parabiosis scheme for producing abnormal retinotectal pathways in the absence of activity (see "Materials and Methods"). A, An optic vesicle is transplanted from a normal $(+/+)$ axolotl embryo to a genetically eyeless $(e / e)$, or in some cases an enucleated normal, embryo. $B$, After the eye primordium has healed into place the animal is rotated to its other side, where a roughly rectangular piece of epidermis is stripped from the mid-flank region. A similar piece of epidermis is stripped off the opposite side of a California newt embryo. $C$, The two embryos in $B$ are pressed together and allowed to heal for several hours. The result is a parabiosis between the newt and the eyeless axolotl with the transplanted eye. Since the newt embryo contains TTX, the axolotl which is sensitive to the toxin is paralyzed. 
(Humphrey, 1969; Van Deusen, 1973; Epp, 1978). Eye primordia including optic stalks were dissected out of normal embryos with a tungsten pin. Wounds were made in the host embryos' epidermis and underlying neuroepithelium, slightly dorsal and posterior to where eyes and stalks would be in normal embryos, in order to introduce abnormal optic nerve entry points. The eyes were transferred to the host, taking care to preserve the normal orientation and handedness of the transplant as well as to mesh the optic stalks with the neuroepithelial wounds. The transplanted eyes were held in place for approximately $1 \mathrm{hr}$ by the weight of small chips of coverglass. In about one-third of the cases, normal embryos, both of whose eyes had been removed (and in fact donated for other hosts), were themselves used as hosts instead of genetically eyeless embryos. These enucleated normal hosts were used both as controls for the genetically eyeless animals and to increase the number of experimental cases.

The second step of the operation consisted of parabiosing the operated axolotl embryos before stage 28 with California newt embryos. A rectangular piece of midflank epidermis was peeled off the axolotl embryo on the side opposite the eye transplant. A similar, mirror-symmetric piece was excised from the newt. The two embryos were then tightly fitted, wound to wound, into a depression in the wax operating dish. They were allowed to heal together overnight. By the next morning the two animals were usually fused. Newt embryos younger than the axolotls were used for parabiosis in order to maximize the concentration and duration of exposure to the toxin which is stored in the newt's yolk granules (Twitty, 1937). All operations were performed in $100 \%$ Steinberg's solution with no anesthetic and double the normal concentration of divalent cations to accelerate healing (Rugh, 1962).

Parabiotic axolotls were checked for mobility and responsiveness to probing at all stages following parabiosis. Previous experiments with parabiotics revealed that, soon after the newt's yolk is used up, the axolotl may recover some neural activity and mobility (Twitty, 1937). The reason for this recovery is unknown. Perhaps the toxin concentration falls as the maternal store is depleted. This may be only part of the explanation, however, since no recovery of impulse activity was noticed in axolotl eyes transplanted to newt embryos, and these newt embryos contained about $10^{-6} \mathrm{M}$ toxin in their tissues (Harris, 1980a). Perhaps catabolic activity in the developing liver of the axolotl twin is also involved in the recovery. In any case, in order to avoid this complication the axolotl twins, besides being continuously monitored, were killed at stages 43 to 45 , several days before recovery. Since retinal fibers arrive in the tectum only shortly before these stages (personal observations; also cf. the closely related Ambystoma tigrinum, stage 39, Herrick, 1941, 1942; and Xenopus laevis, stage 37/38, Holt and Harris, 1983), this provided an additional advantage, in that it meant a relatively early projection pattern was being studied.

Histochemistry. In order to assess the retinal projection from the transplanted eye, the tip of an insect pin coated with horseradish peroxidase (HRP; Sigma Grade VI) was

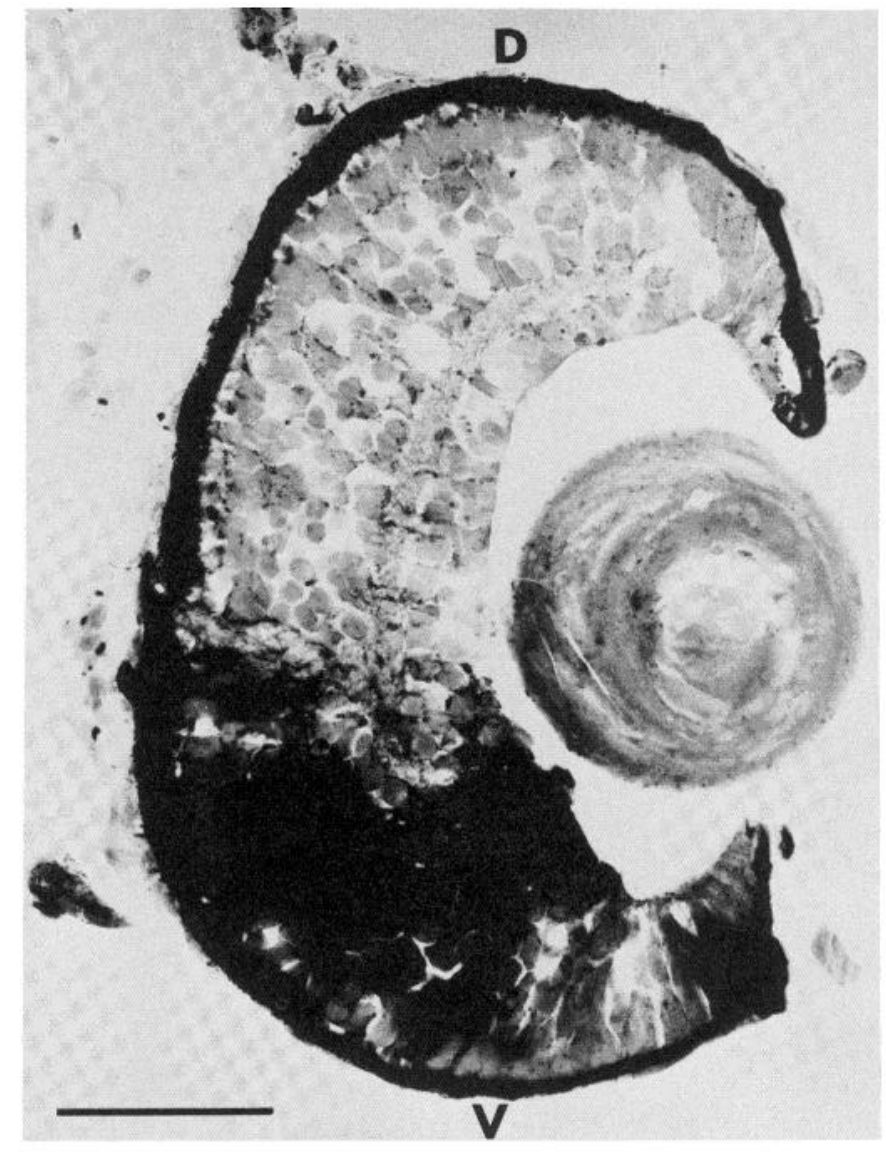

Figure 2. Cross-section through a labeled eye in an experimental animal. In this case the HRP-coated pin was used to label the ventral portion of the retina. Scale bar $=100 \mu \mathrm{m}$.

inserted into the retina, usually in a dorsal or ventral sector (see Fig. 2). The ventral choroidal fissure served as a landmark, and its normal position demonstrated that the eye had been transplanted in the proper orientation. The pin was held there until the HRP dissolved. Two days later the parabiotic twins were anesthetized, their head epidermis was removed, and they were fixed by immersion in a solution of $2.0 \%$ glutaraldehyde in 0.1 M $\mathrm{PO}_{4}$ buffer. After $6 \mathrm{hr}$ of fixation, the animals were transferred into a $25 \%$ sucrose in phosphate buffer solution, and after they'sank they were sectioned at $12 \mu \mathrm{m}$ on a SLEE cryostat. Sections were dried in air on subbed slides and then reacted for HRP according to the methods of Hanker et al. (1977).

Controls. Two sets of controls were run simultaneously. The first was simply a set of age-matched normal axolotls which had undergone no operations. The second were normal axolotls without any eye operations parabiosed to newts.

\section{Results}

Parabiotic animals. Forty experimental parabiotes were constructed. In all but two of these the axolotl twin had one eye which was of donor origin (Fig. 3). The exceptions had three eyes and must have been the result of transplanting an eye to a normal instead of an eyeless host. Twenty-one of the one-eyed animals survived to 


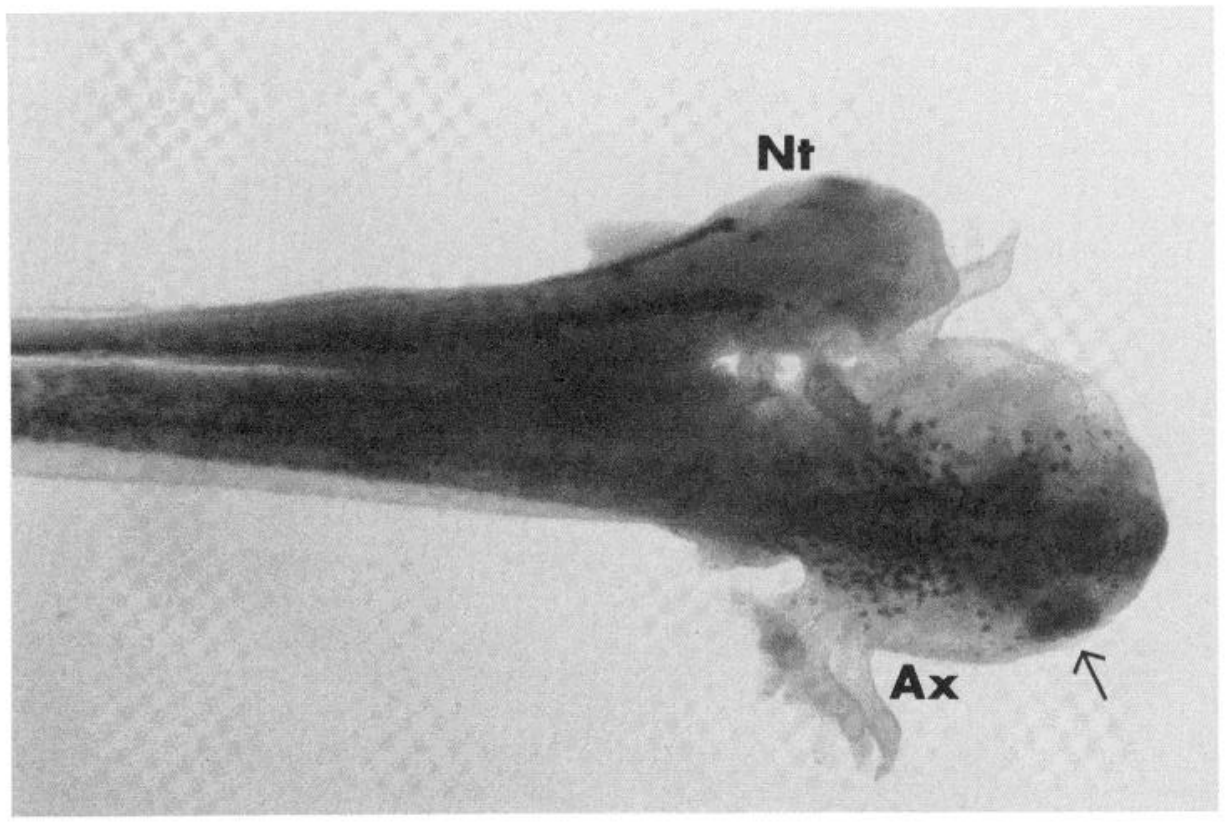

Figure 3. Photograph of a parabiotic axolotl-newt $(A x-N t)$ pair at the time of eye labeling. Note that the axolotl has only one eye (arrow), the transplanted one, on the side opposite the newt.

TABLE I

Summary of results

This table summarizes the basic findings reported in the text. Seventeen of 21 experimental animals showed central projections; 7 of these were to the contralateral tectum, 10 to the ipsilateral. Under "Termination Site in Tectum" ventrolateral is appropriate for a dorsal retina, and dorsomedial is appropriate for a ventral retina. "Spread" means the label was not well restricted to the normal termination zone. Besides the possibility that this was due to inaccurate axonal navigation and synapse formation in these few cases, it is possible that they were false negatives, being the result of poor histology or of the pin hitting the optic nerve head and thus labeling the entire complement of fibers. The overwhelming majority of labeled axons, however, were found to project to their normal termination sites in both the experimental animals and the two sets of controls.

\begin{tabular}{|c|c|c|c|c|}
\hline Part of Retina Labeled & Projection into Host CNS & Contralateral & Ipsilateral & Termination Site in Tectum \\
\hline \multicolumn{5}{|c|}{ Experimental animals } \\
\hline Dorsal $(n=9)$ & 9 & 4 & 5 & Ventrolateral 8, Spread 1 \\
\hline Ventral $(n=8)$ & 6 & 3 & 3 & Dorsomedial 5, Spread 1 \\
\hline \multicolumn{5}{|c|}{ Control series I: Normal axolotls, no parabiosis } \\
\hline Dorsal $(n=4)$ & 4 & 4 & 0 & Ventrolateral 4 \\
\hline Ventral $(n=5)$ & 5 & 5 & 0 & Dorsomedial 5 \\
\hline \multicolumn{5}{|c|}{ Control series II: Normal axolotls, parabiosed to newts } \\
\hline Ventral $(n=1)$ & 1 & 1 & 0 & Dorsomedial 1 \\
\hline
\end{tabular}

the stage of HRP labeling, and the results from these animals and the controls are outlined in Table I.

Touching or pricking the newt twin almost always stimulated swimming or wriggling of that animal, whereas similar prodding, even poking the spinal cord with a pin, produced no response in the axolotl twin at these stages (cf. Twitty, 1937). No axolotl showed signs of sensitivity or movement from the time of parabiosis to the moment of fixation. These results indicate that the parabiosis was effective in eliminating $\mathrm{Na}^{+}$-dependent, TTX-sensitive activity in the axolotl embryo (see previous physiological demonstration, Harris, 1980a).

Central projections. In 17 of the 21 experimental cases studied, retinal fibers from the transplanted eye entered the host CNS. In the remaining four no optic nerve was found. All 17 experimental cases with optic nerves were found to make projections to the host tectum (Figs. 6, 8, and 9 ; Table I). In 10 cases the projection was to the ipsilateral tectum, and in the remaining 7 cases the projection was to the contralateral tectum only. All those projections that were contralateral entered at the chiasm and projected along the normal pathway. Those that projected to the ipsilateral tectum entered the host CNS at ectopic sites (cf. Harris, 1980b, 1982). No cases were found in which labeled retinal fibers entered the CNS and projected to abnormal targets.

Since normal young larval axolotls have only contralateral retinotectal projections, the ipsilateral projections found here formed the most interesting subset of cases, for in these the pathway to the tectum was inherently abnormal. This follows from the fact that the optic nerves penetrated the host brain at ectopic positions. 

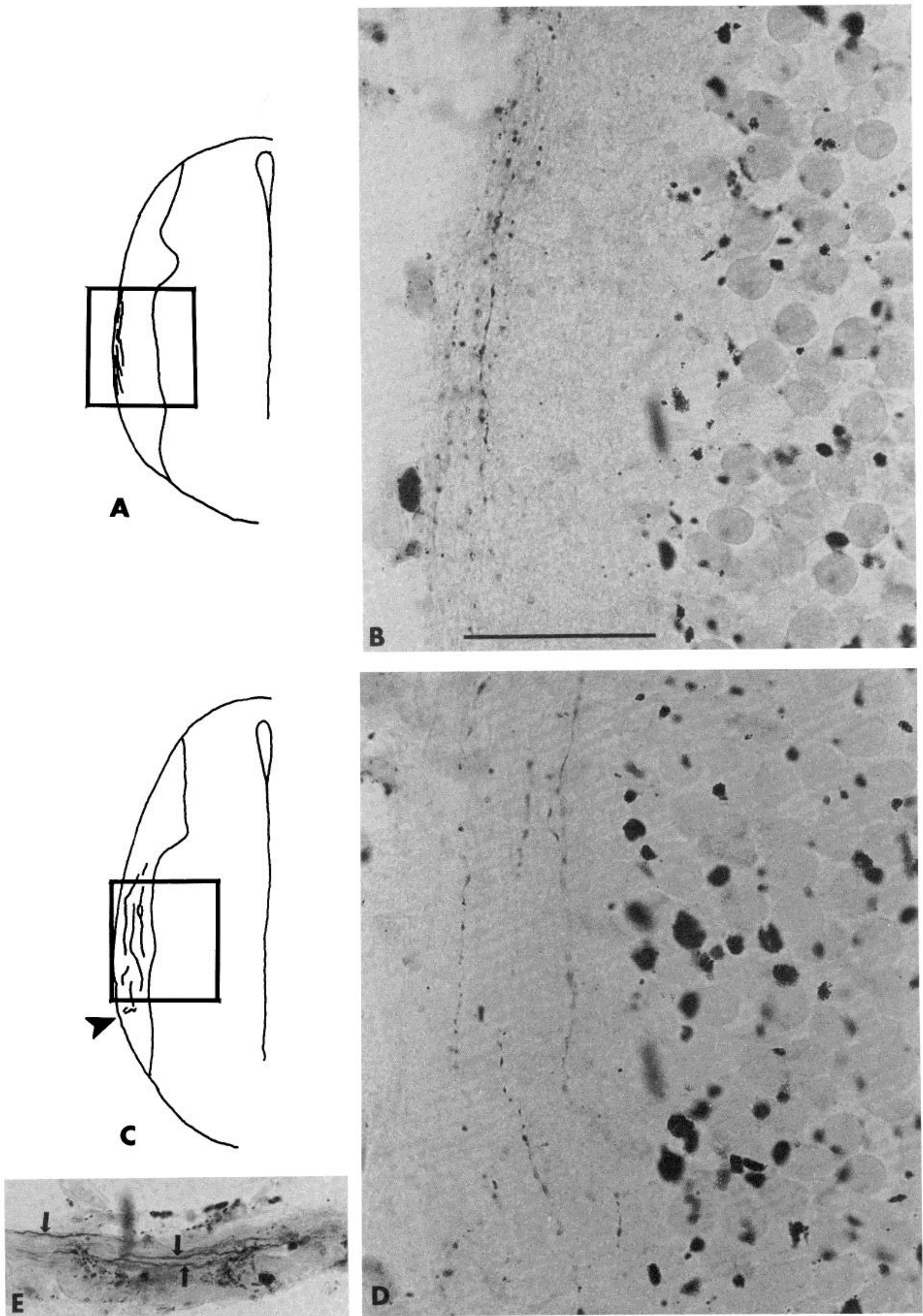

Figure 4. Optic tract in normal and experimental animals (see the text). $A$, Camera lucida drawing of labeled retinal fibers gathered near the pial surface in a normal axolotl. $B$, Photomicrograph of the inset square in $A$ showing some individually labeled axons. Scale bar $=50 \mu \mathrm{m}$ for $B, D$, and $E$. C , Camera lucida drawing of the optic fibers heading toward the tectum in an experimental embryo. The arrowhead points to the optic nerve entry point of the transplanted eye. $D$, Photomicrograph of the inset in $C$ showing that labeled fibers are separated from each other and spread rather evenly between the cellular layer of the brain and the pial surface. This type of aberrant pathway is common in experimental animals, yet these fibers still manage to reach their targets. $E$, Axons labeled in a longitudinal section of an optic nerve from a transplanted eye. Although a cluster of cells was labeled in the retina (see Fig. 3), the labeled axons in the nerve (arrows) are well separated from each other. 
Unless the entry points were directly on what would normally be the optic tract, the initial trajectories of the transplanted optic pathways in these cases must have been abnormal. In addition, in several of these animals the fibers were not obviously fasciculated in the nerve (Fig. 4), and upon penetrating the CNS, they grew rather directly to the tectum along abnormal pathways in the central neuropil of the host CNS rather than along the normal optic tract at the pial surface (Fig. 4). These fibers may have come in contact with the normal optic tract in its final approach to the tectum, but it is clear that the majority of the pathway was abnormal. Although it is difficult to quantitate exactly how abnormal these pathways were, rough estimates can be made. For example, normal axons stayed within about $15 \mu \mathrm{m}$ of the pial surface, whereas abnormal axons ranged as much as $50 \mu \mathrm{m}$ away (see Fig. 4); thus they were at least $35 \mu \mathrm{m}$ off the normal pathway in their mediolateral coordinates. Some ectopic nerves penetrated the brain as far as about $200 \mu \mathrm{m}$ posterior and $250 \mu \mathrm{m}$ dorsal to the normal optic nerve entry point at the chiasm. When these pathways were first formed, the animals were younger and their brains were about $30 \%$ smaller, but this growth was probably largely counterbalanced by shrinkage due to histological preparation. Thus these fibers may have been $100 \mu \mathrm{m}$ or more off their normal pathways as they grew to their targets. Since only small subsets of retinal fibers were labeled in these experiments, it is also difficult to say how many axons actually found the normal pathway. Certainly in many of the cases (e.g., Fig. 4), most of the labeled axons were off the pathway, implying the same for the total population of abnormally routed retinal fibers. In these animals then, optic fibers from a transplanted eye entered the CNS at ectopic locations, traveled along abnormal pathways, were prevented from firing impulses, and yet grew in a directed way to the tectum.

Topography. The topography of the projection was assessed only roughly and was compared to control groups (see Figs. 5 to 9 , Table I). The general result was that labeled fibers from the dorsal retina projected to termination zones in the more ventrolateral tectum, whereas the ventral retinas of experimental animals sent axons to the more dorsomedial tectum. Although the assessment of this topography was necessarily rough, the precision of the projection in both control groups was similar to and certainly not significantly different from that of the experimental group (Fig. 9). Although this mapping procedure only detects the differences between dorsal and ventral retinal projections to the tectum, it

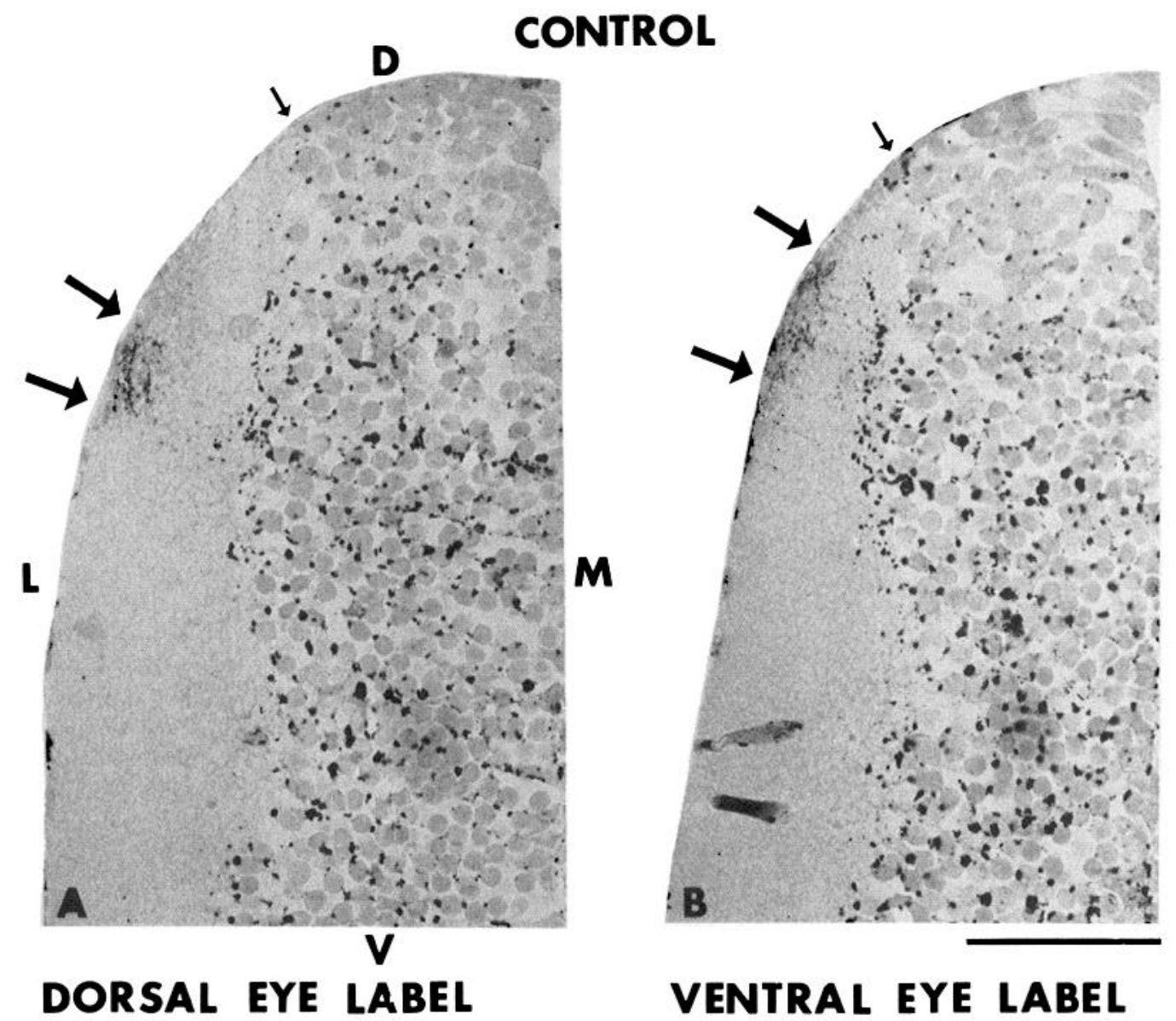

Figure 5. Tectal label in control embryos. In $A$ the dorsal retina was labeled, in $B$ the ventral retina was labeled. The small arrow near the top of each section points to the dorsomedial boundary between the cellular layers and the tectal neuropil which is used as a landmark. The large arrows point to the dorsomedial and ventrolateral extent of the label in the two cases. Notice that in $A$ the label is more ventrolateral with respect to the landmark than in $B$. This indicates normal topography with dorsal retina mapping to ventrolateral tectum and ventral retina mapping to dorsomedial tectum. $D$, dorsal; $V$, ventral; $L$, lateral; $M$, medial. Scale bar $=100 \mu \mathrm{m}$. 

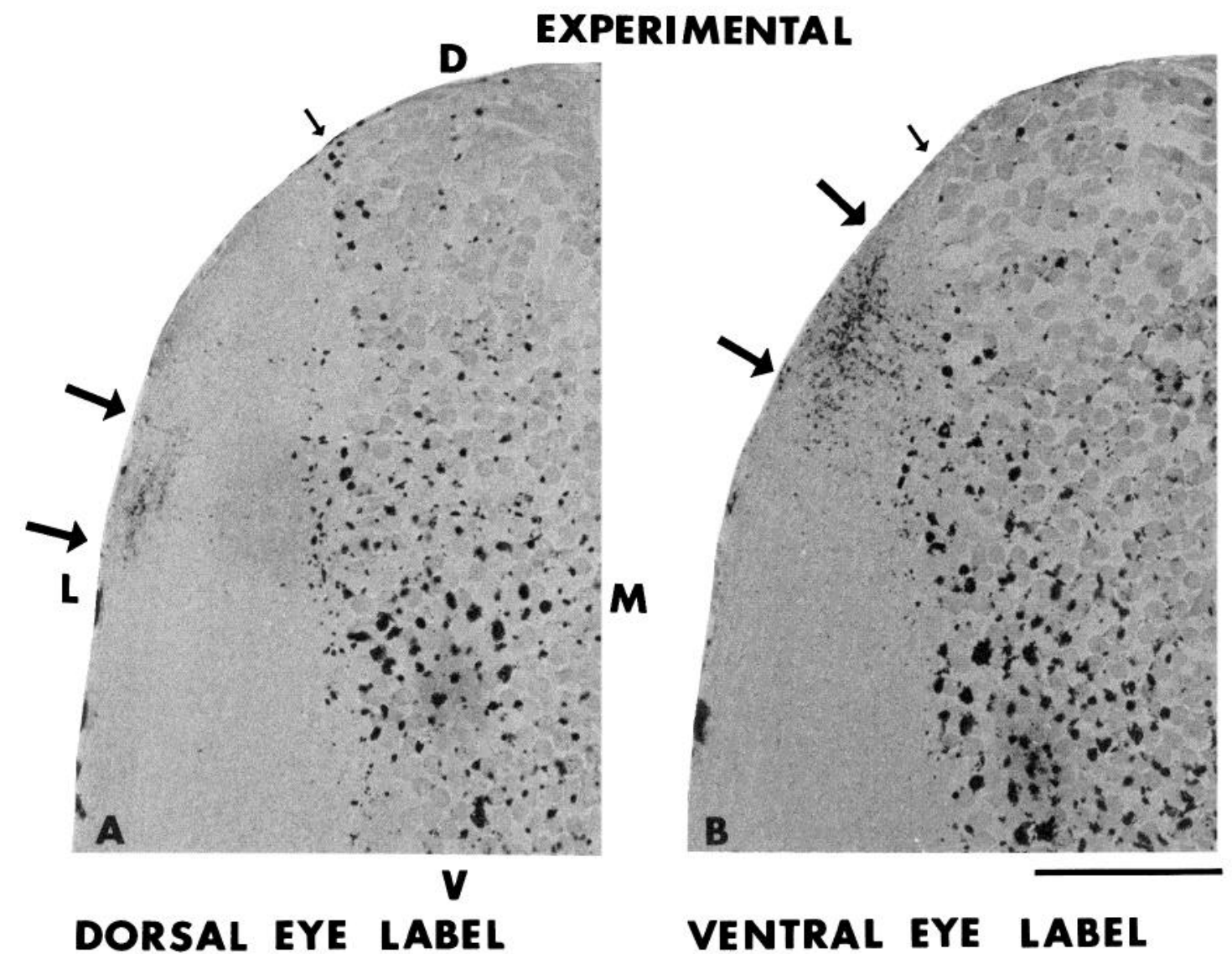

Figure 6. Tectal label in experimental animals. In $A$ the dorsal retina was labeled; in $B$ the ventral retina was labeled. Conventions are as in Figure 5. This result not only shows projections to the tectum in these two animals but also indicates at least grossly normal topography can form along aberrant pathways and in the absence of impulse activity.

may be worth emphasizing that the entire mediolateral extent of the tectum at these stages is only about 250 $\mu \mathrm{m}$, and overlap between dorsal and ventral label is rare. In numerical terms the data suggest a precision of 100 $\mu \mathrm{m}$ or better. Thus, retinal fibers growing along abnormal pathways in the absence of activity not only were able to grow to their normal target structures, but also were able to make a topographic map on it, even at early stages of development.

\section{Discussion}

These experiments show that developing retinal fibers can grow to their appropriate target neuropil and make topographic projections in spite of the fact that these axons have been both forced to travel along abnormal pathways and prevented from firing impulses. Previous experiments have shown that these factors can be manipulated separately without preventing axons from reaching their targets.

The transplantation of axolotl eyes to California newts proved that developing axons in situ could navigate to their proper targets in spite of never firing $\mathrm{Na}^{+}$-mediated action potentials (Harris, 1980a). In these experiments, however, the axons of the host eyes were both insensitive to the toxin and traveling along normal pathways, leaving open the possibility that these host axons play some role in directing the silenced axons to the tectum. Thus, even though the poisoned axons of transplanted axolotl eyes often traveled along aberrant pathways to their newt targets, they may have been guided there by interacting with the normally active and normally coursing axons of the newt's own optic fibers. In the present experiment, this possibility has been eliminated. In the previous axolotl-to-newt study only the retinal fibers-the projecting axons-were silenced. The target, the newt's tectum, was normally active. In the present experiments, on the other hand, both the transplanted retina and the host tectum were sensitive to the toxin since they were both of axolotl origin. Thus it is now clear that $\mathrm{Na}^{+}-$ dependent, TTX-sensitive impulse activity in the postsynaptic target as well as the presynaptic population of fibers is unnecessary in navigation to a proper target. The possibility that $\mathrm{Ca}^{2+}$ spikes are necessary for axonal navigation has yet to be experimentally addressed.

It may be re-emphasized that "proper target" in this case means only a roughly correct topography. It may well be that the map is not as accurate as it would have been in the presence of activity, for in the regenerating retinotectal projection in goldfish, impulse activity is essential for topographic refinement (Schmidt and Edwards, 1982; Meyer, 1983), whereas gross order can appear without activity (Harris, 1980a; Schmidt and Edwards, 1982; Meyer, 1983). Unfortunately, even though the mapping precision demonstrated here seems to be as 


\section{CONTROL}
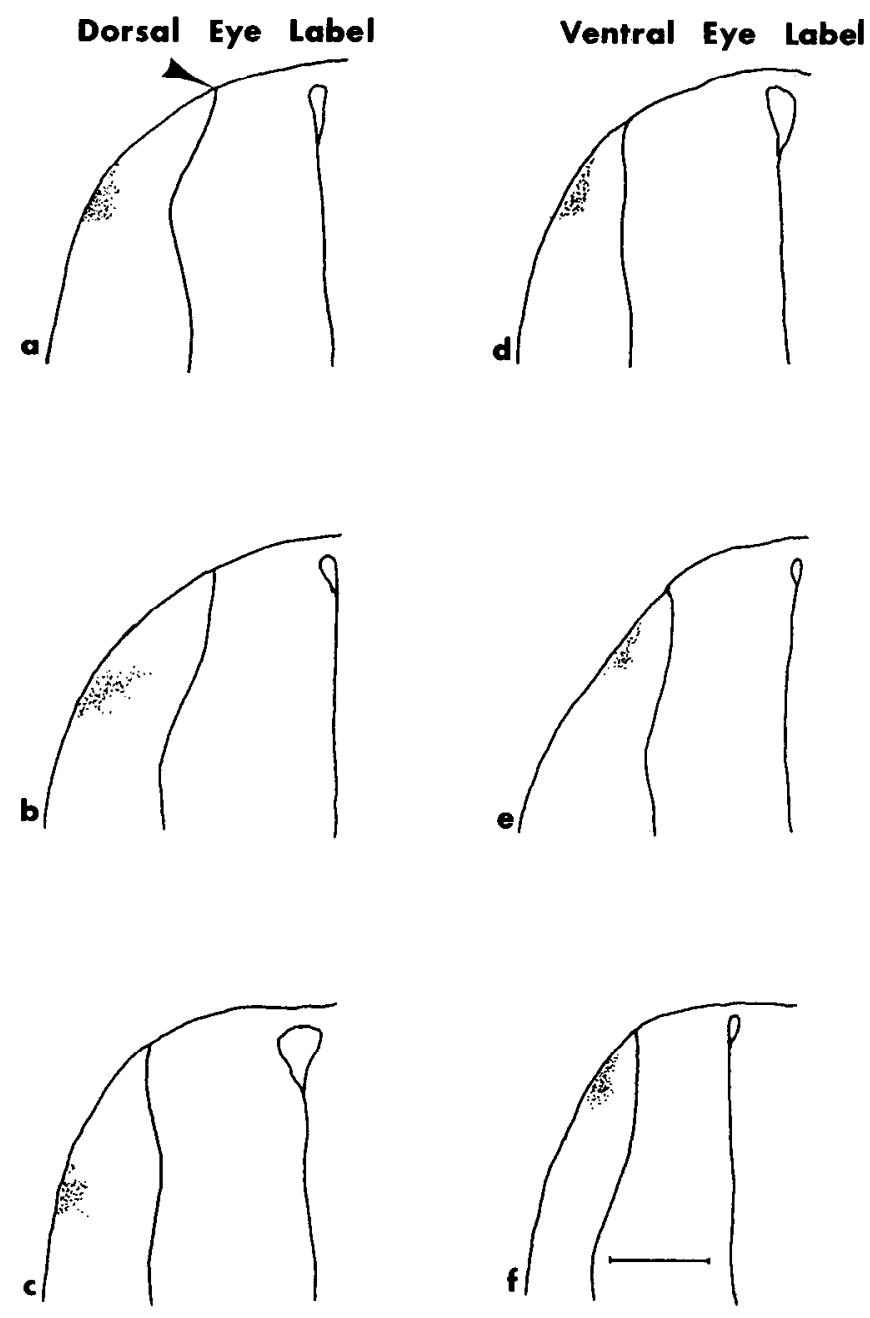

Figure 7. Camera lucida drawings of tectal label in six different control embryos. Those on the left ( $a, b$, and $c$ ) had their dorsal retinas labeled. 'Ihose on the right $(d, e$, and $f$ ) had their ventral retinas labeled. Notice that the ventral retina projects to a more dorsomedial region of the tectum than does the dorsal retina. The arrowhead in a points to the border between the cellular layer and the neuropil (see Figs. 5 and 6). Scale bar = $100 \mu \mathrm{m}$.

good as $100 \mu \mathrm{m}$ or better, the methods of the present study are too crude to assess anything but gross order because the entire tectum is so small at these stages. This leaves the question of the importance of activity in the precision of the developing projection unresolved.

One possibly interesting side issue of the present study is the high incidence of entirely contralateral projections from the transplanted eyes when compared to previous studies. In particular, in a previous study of eyes transplanted to genetically eyeless axolotls, there was no instance of a purely contralateral projection among 21 cases (Harris, 1982). In the present study, 7 of 17 projections were purely contralateral. It is possible that the reason for this difference has to do with impulse activity, which was not inhibited in the first experiment. It is more likely, however, that the difference resulted from the fact that about one-third of the host animals in the present experiments were enucleated normal embryos, whereas in the previous study they were all genetically eyeless. Thus, even though a wound was made dorsal to the attachment site of the normal optic stalk in the neuroepithelium of the genetically normal but eyeless host, the stalk of the transplanted eye primordium may have healed preferentially onto the normal site. The fibers that grew into the CNS in these cases thus entered at the optic chiasm and crossed to the opposite side of the brain, as do fibers from normal eyes. In previous studies, when the optic nerves of transplanted eyes joined the host nerve and entered at the chiasm, a normal contralateral projection was formed (Harris, 1980b). Since records were not kept of which hosts were genetically eyeless and which were enucleated normal embryos, neither of these two possibilities can be formally excluded, and further experiments may be warranted.

Combinatorial experiments on axonal navigation have been done in other systems. The afferents from supernumerary cerci in crickets, for example, grow to their appropriate targets in spite of $(a)$ taking abnormal path-

\section{EXPERIMENTAL}
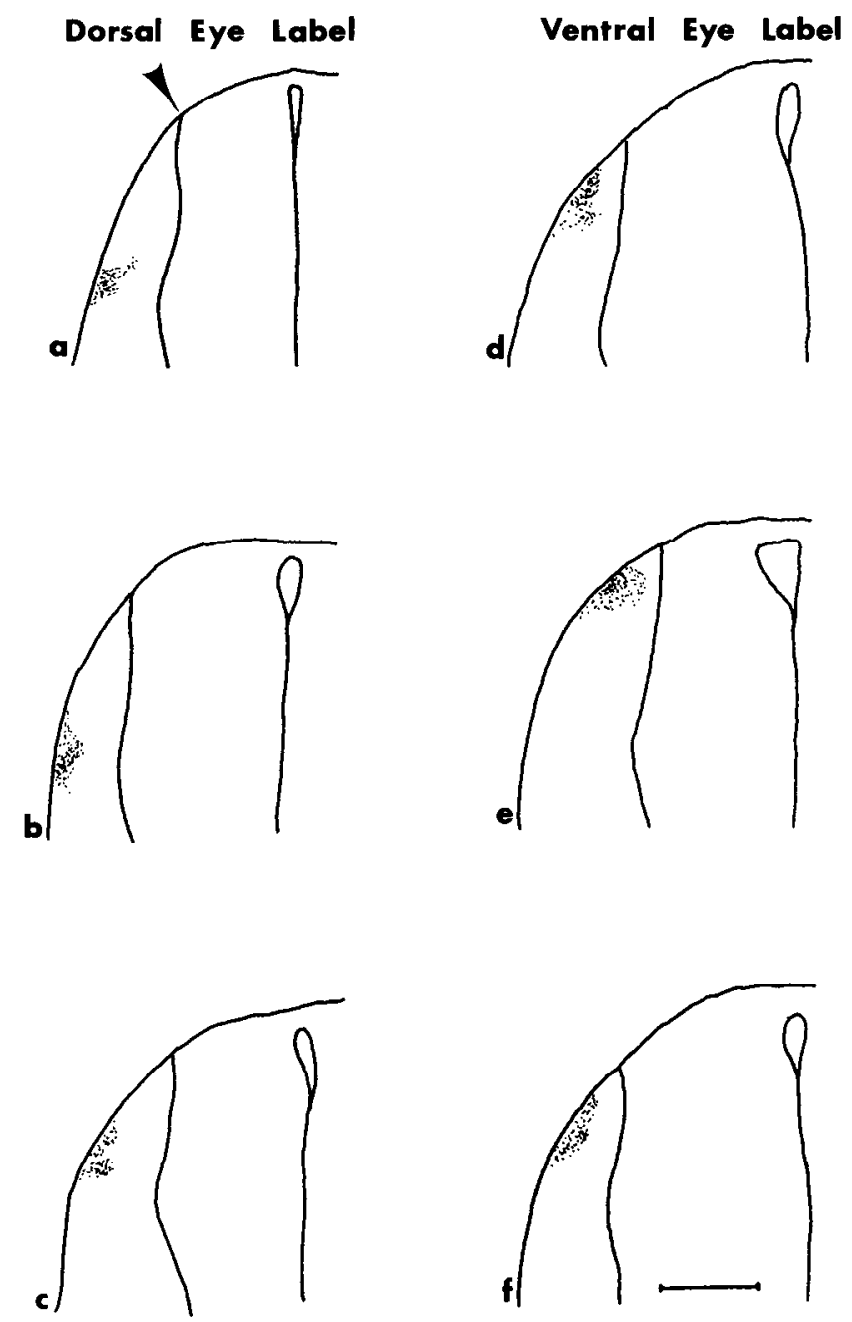

Figure 8. Camera lucida drawings of ipsilateral tectal label in six different experimental embryos. Although the pathway was abnormal and impulse activity was blocked the projection pattern was basically normal (compare to Fig. 7). 


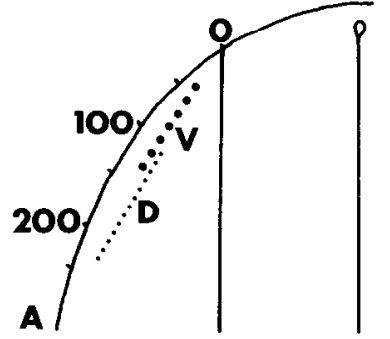

EXPERIMENTAL

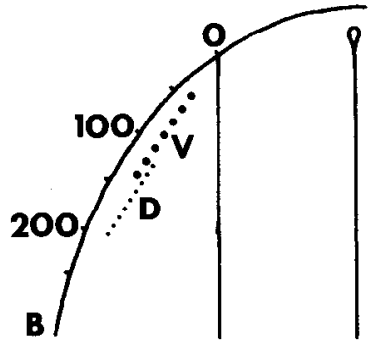

CONTROL

Figure 9. Quantitation of the data for the extent and topography of the projections in experimental $(A)$ and normal $(B)$ animals. Taking the dorsal boundary between the cellular layer and the neuropil as the origin, a scale of micrometers can be drawn down along the pial surface of the tectum. The average boundary extents for ventral eye labels in control animals were: top, $43 \pm 12 \mu \mathrm{m} ;$ bottom, $129 \pm 25 \mu \mathrm{m}(n=5)$, compared to ventral eye labels in ipsilaterally projecting experimental animals (top, $39 \pm 13 \mu \mathrm{m}$; bottom, $133 \pm 14 \mu \mathrm{m}(n=3)$ ). For dorsal eye labels in controls they were: top, $127 \pm 6 \mu \mathrm{m}$; bottom, 197 $\pm 13 \mu \mathrm{m}(n=4)$, and in ipsilaterally projecting experimentals, top, $111 \pm 29 \mu \mathrm{m}$; bottom, $223 \pm 26 \mu \mathrm{m}(n=5)$. Control and experimental results do not differ significantly. Standard errors not included in line segment lengths.

ways, $(b)$ growing into the CNS at later than normal stages, and $(c)$ competing with afferents from normal cerci (Murphey et al., 1983). In another example, the rotation of a piece of the spinal cord in chick embryos forces these axons to grow along abnormal pathways to reach their correct targets and seems to interfere with the normal temporal order of fibers contacting their targets (Lance-Jones and Landmesser, 1980). In this situation, also, fibers often still grow directly to their normal targets. This implies that, in the peripheral nervous system, both factors can be disrupted simultaneously without major consequences for axonal navigation.

Since many mechanisms are implicated to play roles in axonal navigation and the formation of neuronal connections, perhaps there is, as recent models have suggested, a hierarchical set of forces that act on axonal growth cones and terminals guiding them to their targets (Fraser, 1980; Fraser and Hunt, 1980). If one of these forces is eliminated and axons are found still capable of developing appropriate connections, it does not mean that the particular force in question plays no role in axonal navigation. It may simply mean that the other forces can either compensate in the appropriate way or provide redundant information. The obvious way to investigate the possibility that combinations of factors are used by growing axons is to interfere with the proposed factors in a combinatorial way. The result that axons are able to navigate to their targets in the combined absence of normal pathways and impulse activity means that other factors by themselves or in combination are sufficient to guide developing axons to their targets. Such other factors include chemoaffinity mechanisms, timing mechanisms, and fiber-interactive mechanisms. It may be possible to alter an amphibian embryo such that more than two factors are simultaneously disrupted. In fact, due to imprecise matching of donors and hosts (often two stages apart), timing of outgrowth was probably also upset, although this was not examined. If and when the specific molecules involved in chemical guidance come under experimental control, cytochemical forces may also be directly tested, both alone and combinatorially.

\section{References}

Bodick, N., and C. Levinthal (1980) Growing optic nerve fibers follow neighbors during embryogenesis. Proc. Natl. Acad. Sci. U. S. A. 77: 4374-4378.

Bonhoeffer, F., and J. Huf (1982) In vitro experiments on axon guidance demonstrating an anterior-posterior gradient on the tectum. EMBO J. 1: 427-431.

Bunt, S. M., and T. J. Horder (1977) A proposal regarding the significance of simple mechanical events, such as the development of the choroid fissure, in the organization of central visual projections. J. Physiol. (Lond.) 272: 10-12P.

Easter, S. S., Jr. (1983) Postnatal neurogenesis and changing connections. Trends Neurosci. 6: 53-56.

Epp, L. (1978) A review of the eyeless mutant in the Mexican axolotl. Am. Zool. 18: 169-180.

Fawcett, J. W., and R. M. Gaze (1982) Retinotectal fiber pathways from normal and compound eyes in Xenopus. J. Embryol. Exp. Morphol. 72: 19-37.

Feldman, J. D., R. M. Gaze, and M. J. Keating (1971) Delayed innervation of the optic tectum during development in Xenopus laevis. Exp. Brain. Res. 14: 16-23.

Ferguson, B. A. (1983) Simultaneous elimination of normal fiber-fiber interactions and impulse activity does not prevent appropriate tectal innervation. Soc. Neurosci. Abstr., Vol. 9.

Fraser, S. E. (1980) A differential adhesion approach to the patterning of nerve connections. Dev. Biol. 79: 453-464.

Fraser, S. E., and R. K. Hunt (1980) Retinotectal specificity: Models and experiments in search of a mapping function. Annu. Rev. Neurosci. 3: 319-352.

Fujisawa, H., H. Tani, K. Watanabe, and Y. Ibata (1982) Branching of regenerating retinal axons and preferential selection of appropriate branches for specific neuronal connections in the newt. Dev. Biol. 90: 43-57.

Gaze, R. W. (1970) The Formation of Nerve Connections, Academic Press, Inc., London.

Gaze, R. M., and J. W. Fawcett (1982) Pathways of Xenopus optic fibers regenerating from normal and compound eyes under various conditions. J. Embryol. Exp. Morphol. 73: 1738.

Halfter, W., M. Claviez, and U. Scharz (1981) Preferential adhesion of tectal membranes to anterior embryonic chick retinal neurites. Nature 292: 67-70.

Hanker, J. S., P. E. Yates, C. S. Metz, and A. Rustioni (1977) A new specific, sensitive and noncarcinogenic reagent for the demonstration of horseradish peroxidase. Histochem. J. 9: 789-792.

Harris, W. A. (1980a) The effects of eliminating impulse activity on the development of the retinotectal projection in salamanders. J. Comp. Neurol. 194: 303-317.

Harris, W. A. (1980b) Regions of the brain influencing the projection of developing optic tracts in salamander. J. Comp. Neurol. 194: 319-333.

Harris, W. A. (1982) The transplantation of eyes to genetically eyeless salamanders: Visual projections and somatosensory interactions. J. Neurosci. 2: 339-353.

Herrick, C. J. (1941) Development of the optic nerves of Amblystoma. J. Comp. Neurol. 74: 473-534.

Herrick, C. J. (1942) Optic and postoptic systems of the brain of Amblystoma tigrinum. J. Comp. Neurol. 77: 191-353.

Hibbard, E. (1967) Visual recovery following regeneration of 
the optic nerve through the oculomotor root in Xenopus. Exp. Neurol. 19: 350-356.

Holt, C. E. (1983) Does timing of axon outgrowth influence initial retinotectal topography in Xenopus? J. Neurosci. 4: $1130-1152$.

Holt, C. E., and W. A. Harris (1983) Order in the initial retinotectal map in Xenopus: A new technique for labelling growing nerve fibers. Nature 301: 150-152.

Horder, T., and K. A. C. Martin (1978) Morphogenetics as an alternative to chemospecificity in the formation of nerve connections. J. Embryol. Morphol. 46: 147-170.

Humphrey, R. R. (1969) A recently discovered mutant "eyeless" in the Mexican axolotl (Ambystoma mexicanum). Anat. Rec. 163: 306 .

Jacobson, M. (1978) Developmental Neurobiology, Plenum Press, New York.

Keeton, W. T. (1974) The mystery of pigeon homing. Sci. Am. 231: $96-107$.

Lance-Jones, C., and L. Landmesser (1980) Motoneurone projection patterns in the chick hind limb following early partial reversals of the spinal cord. J. Physiol. (Lond.) 302: 581-602.

Letourneau, P. C. (1982) Nerve fiber growth and its regulation by extrinsic factors. In Neuronal Development, N. C. Spitzer, ed., pp. 213-254, Plenum Press, New York.

Meyer, R. L. (1983) Tetrodotoxin inhibits the formation of refined retinotopography in goldfish. Dev. Brain Res. 6: 293298.

Murphey, R. K., S. E. Johnson, and D. S. Sakaguchi (1983) Anatomy and physiology of supernumerary cercal afferents in crickets: Implications for pattern formation. J. Neurosci. 3: $312-325$.

Rugh, R. (1962) Experimental Embryology, Burgess, Minneap olis.
Schmidt, J. T. (1982) The formation of retinotectal projections. Trends Neurosci. 4: 111-116.

Schmidt, J. T., and D. L. Edwards (1982) Activity sharpens the map during the regeneration of the retinotectal projection in goldfish. Soc. Neurosci Abstr. 8: 668.

Schmidt, J. T., C. M. Cicerone, and S. S. Easter (1978) Expansion of the half-retinal projection to the tectum in goldfish: An electrophysiological and anatomical study. J. Comp. Neurol. 177: $257-278$

Scholes, J. H. (1979) Nerve fibre topography in the retinal projection to the tectum. Nature 278: 620-624.

Schreckenburg, S. M., and A. G. Jacobson (1975) Normal stages of development of the axolotl, Ambystoma mexicanum. Dev. Biol. 42: 391-400.

Schwenk, G., and E. Hibbard (1977) An autoradiographic study of optic fiber projections from eye grafts in eyeless mutant axolotls. Exp. Neurol. 55: 498-503.

Sperry, R. W. (1963) Chemoaffinity in the orderly growth of nerve fiber patterns and connections. Proc. Natl. Acad. Sci. U. S. A. 50: 703-710.

Straznicky, C., R. M. Gaze, and M. J. Keating (1981) The development of the retinotectal projections from compound eyes in Xenopus. J. Embryol. Exp. Morphol. 62: 13-35.

Trisler, C. D., M. D. Scheider, and M. Niremberg (1981) A topographic gradient of molecules in retina can be used to identify neuron position. Proc. Natl. Acad. Sci. U. S. A. 78: $2143-2149$.

Twitty, V. C. (1937) Experiments on the phenomenon of paralysis produced by a toxin occurring in Triturus embryos. J. Exp. Zool. 76: 67-104.

Van Deusen, E. (1973) Experimental studies on a mutant gene (e) preventing the differentiation of cye and normal hypothalamus promordia in the axolotl. Dev. Biol. 34: 135-158. 